\title{
Computer Simulation of Stochastic Models with SDE-Solver Software Package
}

\author{
Aleksander Janicki ${ }^{1}$, Adam Izydorczyk ${ }^{2}$, and Przemyslaw Gradalski ${ }^{2}$ \\ 1 Mathematical Institute, University of Wrocław, \\ pl. Grunwaldzki 2-4, 50-384 Wrocław, Poland \\ janicki@math.uni.wroc.pl \\ http://www.im.pwr.wroc.pl/ ${ }^{\sim}$ janicki \\ 2 Institute of Mathematics, Wrocław University of Technology, \\ Wybrzeże Wyspiañskiego 27, 50-370 Wrocław, Poland \\ \{izydorcz, gradal\}@im.pwr.wroc.pl
}

\begin{abstract}
The SDE-Solver software package is our own professional $\mathrm{C}++$ program, designed as a scientific computing tool serving for computer construction of solutions to a wide class of systems of stochastic differential equations (SDEs), including a broad class of diffusions with jumps driven by a number of non-Gaussian random measures. It has been developed as an application to the Windows system and its shareware version is available with a monograph 4 .

In this paper we shortly explain what new ideas have been used in order to get useful statistical and visual information on the time evolution of stochastic processes serving as solutions to such SDEs.

Monte Carlo simulations, statistical estimation methods, approximate numerical algorithms, and modern computer visualization techniques applied together allow for constructing solutions to the wide class of semimartingale stochastic differential equations.

In this way the $S D E$-Solver package (as other scientific computing tools) can be successfully applied in searching for proper and reliable stochastic models of real-life phenomena subject to random non-Gaussian disturbances and in studying their various properties.
\end{abstract}

\section{Introduction}

It is commonly understood that any continuous diffusion process $\{X(t): t \geq 0\}$ with given drift and dispersion coefficients $a=a(t, x)$ and $b=b(t, x)$, can be obtained as a solution of the scalar Itô stochastic differential equation

$$
X(t)=X_{0}+\int_{0}^{t} a(s, X(s)) d s+\int_{0}^{t} b(s, X(s)) d B(s)
$$

where $\{B(t)\}_{t \geq 0}$ stands for the Brownian motion process defined on a probability space $\left(\Omega, \overline{\mathcal{F}}, P,\left\{\mathcal{F}_{t}\right\}_{t \geq 0}\right)$. The solution is a real valued and $\left\{\mathcal{F}_{t}\right\}_{t \geq 0}$-adapted diffusion process $\{X(t): t \geq 0\}$. 
More general stochastic process can be described as a solution to the following stochastic differential equation

$$
\begin{aligned}
X(t)=X_{0} & +\int_{0}^{t} a(s, X(s-)) d s+\int_{0}^{t} b(s, X(s-)) d L_{\alpha, \beta}(s) \\
& +\int_{0}^{t} c(s, X(s-)) d \tilde{N}_{\lambda}(s) ; \quad t \geq 0
\end{aligned}
$$

driven by $\alpha$-stable Lévy process $\left\{L_{\alpha, \beta}(t): t \geq 0\right\}$ (see e.g. [2] and [3]), and by compensated Poisson process $\left\{\widetilde{N}_{\lambda}(t): t \geq 0\right\}$ (see [4]).

Both types of stochastic equations described above are special cases of general stochastic differential equations driven by semimartingales, i.e. equations in the following form

$$
X(t)=X_{0}+\int_{0}^{t} f(X(s-)) d Y(s) ; \quad t>0, \quad X(0)=X_{0},
$$

where $\{Y(t)\}$ stands for a given semimartingale process (for the theorems on existence and uniqueness of solutions consult e.g. Protter $[8]$ and the bibliography therein).

In this paper we briefly demonstrate a necessity of application of SDE (2) and systems of SDEs presented below in a vector equation (4) to stochastic modelling of real-life phenomena, and at the same time we try to show the usefulness of the SDE-Solver package, choosing as an example a competing species problem well known in biology. The vast variety of such examples is considered in 3 and [4. One of our latest works, i.e. 6, is related to the construction of optimal portfolio of an investor in a stochastic financial market.

\section{A Class of SDEs Solvable with the SDE-Solver Software Package}

The user friendly $S D E$-Solver package provides the user with computer produced solutions to a wide class of SDEs with given Cauchy type initial conditions. This means that the package offers information on time evolution of the $n$-dimensional stochastic process

$$
\mathbf{X}=\left\{\mathbf{X}(t): t \in\left[t_{0}, T\right]\right\}=\left\{\left(X_{1}(t), \ldots, X_{n}(t)\right): t \in\left[t_{0}, T\right]\right\}
$$

solving the $n$-dimensional SDE, written in the following integral form

$$
\begin{aligned}
\mathbf{X}(t)=\mathbf{X}_{0} & +\int_{t_{0}}^{t} \mathbf{a}(s, \mathbf{X}(s)) d s+ \\
& +\int_{t_{0}}^{t} \mathbf{b}(s, \mathbf{X}(s-)) d \mathbf{L}_{\bar{\alpha}, \bar{\beta}}(s)+ \\
& +\int_{t_{0}}^{t} \mathbf{c}(s, \mathbf{X}(s-)) d \tilde{\mathbf{N}}_{\bar{\lambda}}(s) ; \quad t \in\left[t_{0}, T\right]
\end{aligned}
$$


with initial conditions defined by the vector of $n$ deterministic or random components

$$
\mathbf{X}_{0}=\left(X_{0,1}, \ldots, X_{0, n}\right)
$$

Functional coefficients of (4) are of the following form

$$
\begin{aligned}
& \mathbf{a}: \mathbb{R}_{+} \times \mathbb{R}^{n} \longmapsto \mathbb{R}^{n}, \\
& \mathbf{b}: \mathbb{R}_{+} \times \mathbb{R}^{n} \longmapsto \mathbb{R}^{n \times m_{1}}, \\
& \mathbf{c}: \mathbb{R}_{+} \times \mathbb{R}^{n} \longmapsto \mathbb{R}^{n \times m_{2}},
\end{aligned}
$$

what means that they are functions defined on $\mathbb{R}_{+} \times \mathbb{R}^{n}$ with vector or matrix values, and $\mathbf{L}_{\bar{\alpha}, \bar{\beta}}=\mathbf{L}_{\bar{\alpha}, \bar{\beta}}(t)$ and $\widetilde{\mathbf{N}}_{\bar{\lambda}}=\widetilde{\mathbf{N}}_{\bar{\lambda}}(t)$ are $m_{1}$-dimensional and $m_{2}$-dimensional stochastic processes. Here, for given vectors $\bar{\alpha} \stackrel{\mathrm{df}}{=}\left(\alpha_{1}, \ldots, \alpha_{m_{1}}\right)$, $\bar{\beta} \stackrel{\text { df }}{=}\left(\beta_{1}, \ldots, \beta_{m_{1}}\right)$ of parameters $\alpha_{j}, \beta_{j}$, we have corresponding vector valued stochastic processes

$$
\mathbf{L}_{\bar{\alpha}, \bar{\beta}}(t) \stackrel{\mathrm{df}}{=}\left(L_{\alpha_{1}, \beta_{1}}(t), \ldots, L_{\alpha_{j}, \beta_{j}}(t), \ldots, L_{\alpha_{m_{1}}, \beta_{m_{1}}}(t)\right)
$$

where $\left\{L_{\alpha_{j}, \beta_{j}}\right\}$, for $j=1, \ldots, m_{1}$, constitute a set of $m_{1}$ independent scalar $\alpha_{j}$-stable Lévy processes, with $\alpha_{j} \in(0,2], \beta_{j} \in[-1,1]$. Similarly, a vector $\bar{\lambda} \stackrel{\mathrm{df}}{=}\left(\lambda_{1}, \ldots, \lambda_{m_{2}}\right)$ stands for a vector valued process

$$
\tilde{\mathbf{N}}_{\bar{\lambda}}(t)=\left(\tilde{N}_{\lambda_{1}}(t), \ldots, \widetilde{N}_{\lambda_{j}}(t), \ldots, \tilde{N}_{\lambda_{m_{2}}}(t)\right)
$$

with $m_{2}$ independent components in a form of scalar Poisson processes $\widetilde{N}_{\lambda_{j}}$ with given intensities $\lambda_{j}>0$.

In basic mathematical theoretical textbooks (see e.g. [8] and the literature cited there and in 3]) the general semimartingale stochastic differential equation is considered.

Such equation is written in a shortened general form

$$
d \mathbf{X}(t)=\mathbf{f}(\mathbf{X}(t-)) d \mathbf{Z}(t) ; \quad \mathbf{X}(0)=\mathbf{X}_{0}
$$

where $\mathbf{Z}(t)=\{Z(t)\}_{t \geq 0}$ stands for a given (vector valued) semimartingale. Our family of systems of SDEs, defined by (4), can be interpreted as a (quite important) subfamily of general semimartingale SDEs. It is enough to construct a vector function

$$
\mathbf{f}: \mathbb{R}^{n+1} \longmapsto \mathbb{R}^{(n+1) \times\left(1+m_{1}+m_{2}\right)},
$$

properly placing function parameters a, b, c from (4) in the definition of $\mathbf{f}$. In this way we get immediately theorems on existence and uniqueness of solutions and even appropriate theorems on convergence of some numerical discrete methods (but not on the speed of convergence). Computer construction of approximate solutions to still wider class of stochastic differential equations driven by general Lévy processes and some problems related to convergence of proposed methods are discussed in [5]. 


\section{$3 \quad$ Numerical Approximation of SDEs with Jumps}

For simplicity we will consider here only the scalar SDE (4), i.e. we assume that $n=1$. We believe that it is quite obvious how to extend numerical schemes presented here to the case of systems of SDEs of the form (4) with $n>1$.

We have

$$
\begin{aligned}
X(t)=X_{0} & +\int_{t_{0}}^{t} a(s, X(s)) d s+\sum_{j=1}^{m_{1}} \int_{t_{0}}^{t} b_{j}(s, X(s-)) d L_{\alpha_{j}, \beta_{j}}(s)+ \\
& +\sum_{l=1}^{m_{2}} \int_{t_{0}}^{t} c_{k}(s, X(s-)) d \widetilde{N}_{\lambda_{k}}(s) ; \quad t \in\left[t_{0}, T\right] .
\end{aligned}
$$

By numerical approximation or discretization of a given stochastic process $\mathbf{X}=\left\{X(t): t \in\left[t_{0}, T\right]\right\}$ we understand here a finite family of explicitly known random variables $\left\{X_{i}^{\tau}: i=0,1, \ldots, I\right\}$, such that $X_{i}^{\tau} \approx X\left(t_{i}\right)$ on a given grid of $I+1$ points $t_{i}=t_{0}+i \tau$, for $\tau=\left(T-t_{0}\right) / I$.

The Euler schema for (6) can be written in the following form

$$
\begin{aligned}
X_{i+1}^{\tau}=X_{i}^{\tau} & +\left(a\left(t_{i}, X_{i}^{\tau}\right)-\sum_{l=1}^{m_{2}} \lambda_{l} c_{l}\left(t_{i}, X_{i}^{\tau}\right)\right) \tau+ \\
& +\sum_{l=1}^{m_{1}} b_{l}\left(t_{i}, X_{i}^{\tau}\right) \tau^{1 / \alpha} U_{i, l}+\sum_{l=1}^{m_{2}} c_{k}\left(t_{i}, X_{i}^{\tau}\right) V_{i, l},
\end{aligned}
$$

where for $i=0,1, \ldots, I-1$ the sequences $\left\{U_{i, l}\right\}$ and $\left\{V_{i, l}\right\}$ stand for sequences of independent random variables such that $U_{i, l} \sim \mathcal{N}(0,1)$ or $U_{i, l} \sim S_{\alpha, \beta}(1,0)$, for $l=1,2, \ldots, m_{1}$, and $V_{i, l}$, for $l=1,2, \ldots, m_{2}$, denotes a random variable counting the number of jumps of the process $\widetilde{N}_{\lambda_{l}}$ on the interval $\left[t_{i}, t_{i-1}\right)$.

The computer techniques of visualization of a single trajectory of a solution in jumps to (6) consists simply in plotting points $\left\{\left(t_{i}, \bar{X}_{i}^{\tau}\right): i=0, \ldots, I-1\right\}$

$$
\begin{aligned}
\bar{X}_{i+1}^{\tau}=\bar{X}_{i}^{\tau} & +\left(a\left(t_{i}, \bar{X}_{i}^{\tau}\right)-\sum_{l=1}^{m_{2}} \lambda_{l} c_{l}\left(t_{i}, \bar{X}_{i}^{\tau}\right)\right) \tau+ \\
& +\sum_{j=1}^{m_{1}} b_{j}\left(t_{i}, \bar{X}_{i}^{\tau}\right) \tau^{1 / \alpha} \bar{U}_{i, j}\left(\omega_{0}\right)+\sum_{l=1}^{m_{2}} c_{k}\left(t_{i}, \bar{X}_{i}^{\tau}\right) \bar{V}_{i, l}\left(\omega_{0}\right)
\end{aligned}
$$

where $\bar{U}_{i, j}\left(\omega_{0}\right)$ and $\bar{V}_{i, l}\left(\omega_{0}\right)$ denote computed realizations of random variables $U_{i, j}=U_{i, j}(\omega)$ and $V_{i, j}=V_{i, j}(\omega)$ obtained via Monte Carlo simulation methods.

It is clear now that the solution to (6) can be obtained by an explicite discrete time difference scheme

$$
X_{i+1}^{\tau}=\mathcal{F}\left(t_{i}, X_{i}^{\tau},\left\{U_{i, j}\right\}_{j=1}^{m_{1}},\left\{V_{i, l}\right\}_{l=1}^{m_{2}}\right),
$$

where $i=0,1, \ldots, I-1$. 
After constructing pseudo random samples

$$
\overline{\mathbf{U}}_{i}^{\tau} \stackrel{\mathrm{df}}{=}\left\{\left\{\bar{U}_{i, j}^{\tau}\left(\omega_{k}\right)\right\}_{k=1}^{K}\right\}_{j=1}^{m_{1}}, \quad \overline{\mathbf{V}}_{i}^{\tau} \stackrel{\mathrm{df}}{=}\left\{\left\{\bar{V}_{i, l}^{\tau}\left(\omega_{k}\right)\right\}_{k=1}^{K}\right\}_{l=1}^{m_{2}},
$$

from known probability laws corresponding to random variables $\left\{U_{i, j}\right\},\left\{V_{i, l}\right\}$, for $i=0,1, \ldots, I-1, j=1,2, \ldots, m_{1}, l=1,2, \ldots, m_{2}$ we are in a position to get $I$ random samples $\left\{\left\{\bar{X}_{i}^{\tau}\left(\omega_{k}\right)\right\}_{k=1}^{K}\right\}_{i=0}^{I}$ of a size $K$ from unknown laws of random variables $X_{i}^{\tau}, i=0,1, \ldots, I$.

By a natural analogy with (9), in a framework of SDE (6) we get an iterative obvious formula

$$
\left\{\bar{X}_{i+1}^{\tau}\left(\omega_{k}\right)\right\}_{k=1}^{K}=\mathcal{F}\left(t_{i},\left\{\bar{X}_{i}^{\tau}\left(\omega_{k}\right)\right\}_{k=1}^{K}, \overline{\mathbf{U}}_{i}^{\tau}, \overline{\mathbf{V}}_{i}^{\tau}\right)
$$

for a sequence of random samples $\left\{\bar{X}_{i}^{\tau}\left(\omega_{k}\right)\right\}_{k=1}^{K}$, statistically approximating unknown values $X\left(t_{i}\right)$ of a solution $\mathbf{X}$.

\section{Qualitative and Quantitative Analysis of Time Evolution of Stochastic Processes}

Now we describe briefly computer methods developed in our software package SDE-Solver. Most of them are not in a common usage when solving SDEs even in a classical Itô form. Here we present much more on the topic than one can find in any textbook (see e.g. 7] for comparison) or in available literature on application of computer methods in stochastic modelling.

In order to simplify the presentation we assume that an unknown stochastic process $\mathbf{X}=\left\{X(t): t \in\left[t_{0}, T\right]\right\}$ is to be approximated with a family of random variables $\left\{X_{i}^{\tau}\right\}_{i=1}^{I}$, and further on by a family of pseudo random samples

$$
\left\{\left\{\bar{X}_{i}^{\tau}\left(\omega_{k}\right)\right\}_{k=1}^{K}\right\}_{i=0}^{I}
$$

as is explained in Sect. 3 .

Now we show how to get qualitative and quantitative information on a time evolution of the process $\mathbf{X}$.

\subsection{Visualization of Trajectories}

The common technique of visualization of any stochastic process is a graphical construction of its single approximate trajectory. It is enough to fix properly a value of a parameter $I=I_{r}$, compute $\left(t_{i}, \bar{X}_{i}^{\tau}\left(\omega_{k}\right)\right)$ for $i=0,1, \ldots, I_{r}$, and chose $\omega_{k} \in \Omega$. Finally, joining neighbour points by strait lines we get an approximate graph, appropriate in the case of a continuous trajectories of $\mathbf{X}$, as it is correct for Itô diffusions. For jump diffusions it is necessary to notify in some way the jumps of trajectories. This important in case of solutions to (6). In our package it is possible to produce pictures of many trajectories on a given figure. 


\subsection{Approximate Quantile Lines}

The notion of a $p$-quantile line does not appear in an available statistical literature in a context of approximate construction of solutions to SDEs.

For a fixed $p \in(0,1)$ and a given stochastic process $\mathbf{X}=\left\{X(t): t \in\left[t_{0}, T\right]\right\}$ by a $p$-quantile line we understand a deterministic function $q_{p}=q_{p}(t)$ on an interval $\left[t_{0}, T\right]$ such that

$$
\mathbb{P}\left\{X(t) \leq q_{p}(t)\right\}=p ; \quad t \in\left[t_{0}, T\right]
$$

Computer construction of such function can be reduced to the estimation of a $p$-quantile for all or some of ordered random samples $\left\{\left\{\bar{X}_{i,(k)}^{\tau}\right\}_{k=1}^{K}\right\}_{i=0}^{I}$. In this way we get numbers

$$
q_{p}^{\tau}(i) \approx q_{p}\left(t_{i}\right)
$$

for all $i=0,1, \ldots, I$ or for some subsequence of available values of $i$.

Joining pairs of points $\left(t_{i-1}, q_{p}^{\tau}(i-1)\right),\left(t_{i}, q_{p}^{\tau}(i)\right)$ for $i=1,2, \ldots, I$, we get a graph $q_{p}^{\tau}=q_{p}^{\tau}(t)$ approximating unknown $p$-quantile line $q_{p}=q_{p}(t)$ on the interval $\left[t_{0}, T\right]$, i.e. a visualization of time evolution of a $p$-quantile of $\mathbf{X}$.

\subsection{Estimation of Time Evolution of Densities}

Specialists in stochastic modelling agree that the time evolution of densities $\left\{f_{t}=f(t ; x): x \in \mathbb{R}\right\}_{t \in\left[t_{0}, T\right]}$ of a process $\mathbf{X}=\left\{X(t): t \in\left[t_{0}, T\right]\right\}$, or, simply, a function $f=f(t ; x) \stackrel{\text { df }}{=} f_{t}(x)$, is often an important factor in scientific investigation of stochastic models described by SDEs of different kinds.

In the case of Itô diffusions densities $f=f(t, x)$ can be theoretically obtained from the Fokker-Planck-Kolmogorov partial differential equation of parabolic type. For diffusions with jumps - theoretically and practically - the problem is much more complicated.

When we have to our disposal a family $\left\{\left\{\bar{X}_{i}^{\tau}\left(\omega_{k}\right)\right\}_{k=1}^{K}\right\}_{i=0}^{I}$ of random samples from unknown probability laws, we can apply to each of them one of well known nonparametric estimation of densities method, e.g. an efficient in most cases Rosenblatt-Parzen method. It means that we are able to compute

$$
f_{l, j}=\frac{1}{K} \sum_{k=1}^{K} \frac{1}{b_{K_{l}}} \Phi\left(\frac{x_{j}-\bar{X}_{i_{l}, k}^{\tau}}{b_{K_{l}}}\right)
$$

in order to get a good approximation

$$
f_{l, j} \approx f\left(t_{l}, x_{j}\right)
$$

of values of unknown densities $f_{t_{l}}(x)$ of random variables $X\left(t_{l}\right)$, on a finite grid of points $\left(t_{l}, x_{j}\right)$, for $l=1,2, \ldots, L, j=1,2, \ldots, J$.

This is enough to produce in this way three-dimensional nice approximate pictures of $f=f(t, x)$ with appropriate computer graphics tools at hand. 


\subsection{Estimation of Time Evolution of Entropies}

Having to our disposal a set of numbers $\left\{f_{l, j}\right\}$ coming from (14) we can construct an entropy function, which can be defined in the following way

$$
\mathrm{H}(t) \stackrel{\mathrm{df}}{=}-\int_{-\infty}^{\infty} f(t, x) \log f(t, x) d x .
$$

It is enough to compute numerically integrals

$$
\mathrm{H}_{l} \approx \mathrm{H}\left(t_{l}\right)
$$

and finally to produce an approximate graph of an unknown function $\mathrm{H}=\mathrm{H}(t)$.

\subsection{Functional of a Stochastic Process}

An useful tool for investigation of stochastic models arises from the formula

$$
F(t) \stackrel{\text { df }}{=} \mathbb{E}[h(X(t))] ; \quad t \in\left[t_{0}, T\right] .
$$

All values of this deterministic function on $\left[t_{0}, T\right]$ are determined through the given properly chosen function $h=h(x)$, as expected values of random variables $h(X(t))$. As a special case one can get a time evolution of moments of $\mathbf{X}$, but we should remember that quite commonly diffusions with jumps have no finite moments, e.g. diffusions driven by $\alpha$-stable Lévy motion have no finite moments of order greater then 1 .

In a similar way as before, it is enough to make use of a very simple estimator

$$
F\left(t_{l}\right) \approx \frac{1}{K} \sum_{k=1}^{K} \bar{X}_{i_{l}, k}^{\tau}
$$

for $l=1,2, \ldots, L$.

\subsection{Approximation of Wiener Cylindrical Measures}

Also a notion of Wiener cylindrical measures does not appear in an available literature in a context of approximate construction of solutions to SDEs.

If we fix a set of points $t_{i_{l}}$ from an interval $\left[t_{0}, T\right]$ and a set of corresponding intervals $\left[a_{l}, b_{l}\right]$, then we can ask for values of the following expressions

$$
\mathbb{P}\left\{X\left(t_{i_{l}}\right) \in\left[a_{l}, b_{l}\right]: l=1,2, \ldots, L\right\}
$$

or

$$
\mathbb{P}\left\{X\left(t_{i_{l}}\right) \in\left[a_{l}, b_{l}\right]\right\}, \quad l=1,2, \ldots, L,
$$

known as a Wiener cylindrical measure of a product $\left[a_{1}, b_{1}\right] \times \ldots \times\left[a_{L}, b_{L}\right]$.

When we have to our disposal a set of approximate trajectories of $\mathbf{X}$, large enough, than in an obvious way we are able to compute frequencies of trajectories of $\mathbf{X}$ satisfying such conditions as above. This method provides us with acceptable approximate values of the probabilities from (19) and (20). 


\subsection{Examples of Stochastic Differential Equations}

The SDE-Solver package can serve as a powerfull tool for construction and analyzis of stochastic models describing real-life problems coming from such fields as mathematical and statistical physics, chemistry, biology, engineering, stochastic mathematical finance, etc. (see e.g. 1], [3], [7, 11]). In [3] a variety of examples of results of computer simulations with the use of our methods and previous versions of the $S D E$-Solver package can also be found.

Here we present in more detail an interesting example of a system of two stochastic equations coming from biology.

\section{Volterry-Lotka Equation as a Stochastic Model of Competing Species}

Here we present the two-dimensional stochastic generalization of the deterministic Volterry-Lotka model of competing species, which can be described by the following Gauss-Poisson system of SDEs

$$
\begin{aligned}
X(t)=X_{0} & +\int_{0}^{t} X(s)\left(a_{1}+b_{11} X(s)+b_{12} Y(s)\right) d s+ \\
& +\sigma_{1} \int_{0}^{t} X(s) d B_{1}(s)+ \\
& +\nu_{1} \int_{0}^{t} X(s) d N_{\lambda_{1}}(s) \\
Y(t)=Y_{0} & +\int_{0}^{t} Y(s)\left(a_{2}+b_{21} X(s)+b_{22} Y(s)\right) d s+ \\
& +\sigma_{2} \int_{0}^{t} Y(s) d B_{2}(s)+ \\
& +\nu_{2} \int_{0}^{t} Y(s) d N_{\lambda_{2}}(s) .
\end{aligned}
$$

For computer simulations we fixed the following values of parameters of a system of equations: $a_{1}=3, b_{11}=-1, b_{12}=-2, a_{2}=2, b_{21}=-1, b_{22}=-1$.

For parameters describing random measures in our system of 2 SDEs we have found: $\sigma_{1}=0.05, \sigma_{2}=0.05, \nu_{1}=-0.5, \lambda_{1}=0.1, \nu_{2}=0.25, \lambda_{2}=0.1$.

Initial values are the most interesting for $X(0)=1, Y(0)=1$.

The Gauss-Poisson model with jumps (21) with these fixed values of parameters is favorable for the second species, 'offering' for it an explosive growth $\left(\nu_{2}>0\right)$ in random times, while the first species suffers serious sudden decline in number $\left(\nu_{1}<0\right)$ from time to time. Precisely, in our semimartingale model we get

$$
\mathbb{P}\{X(t) \in[0.5,3.5]\}=\mathbb{P}\{Y(t) \in[-0.3,0.3]\} \approx 0.832, \quad \text { for } t \geq 30,
$$




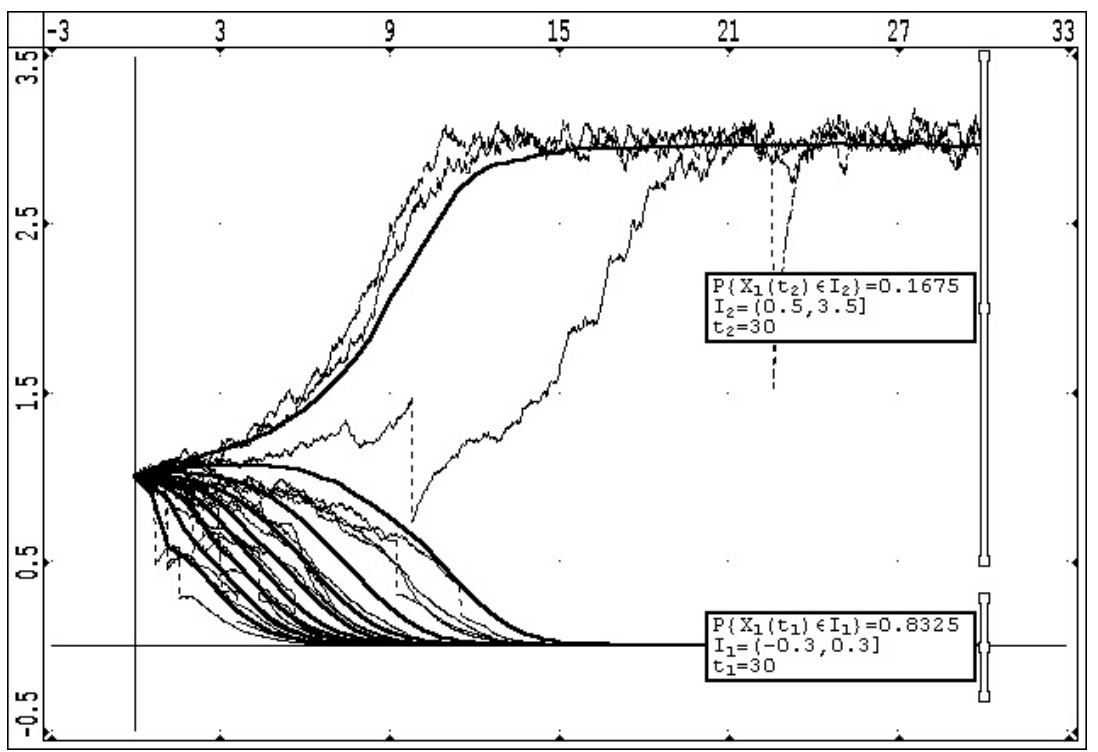

Fig. 1. Stochastic component $X(t)$ of the solution to (21)

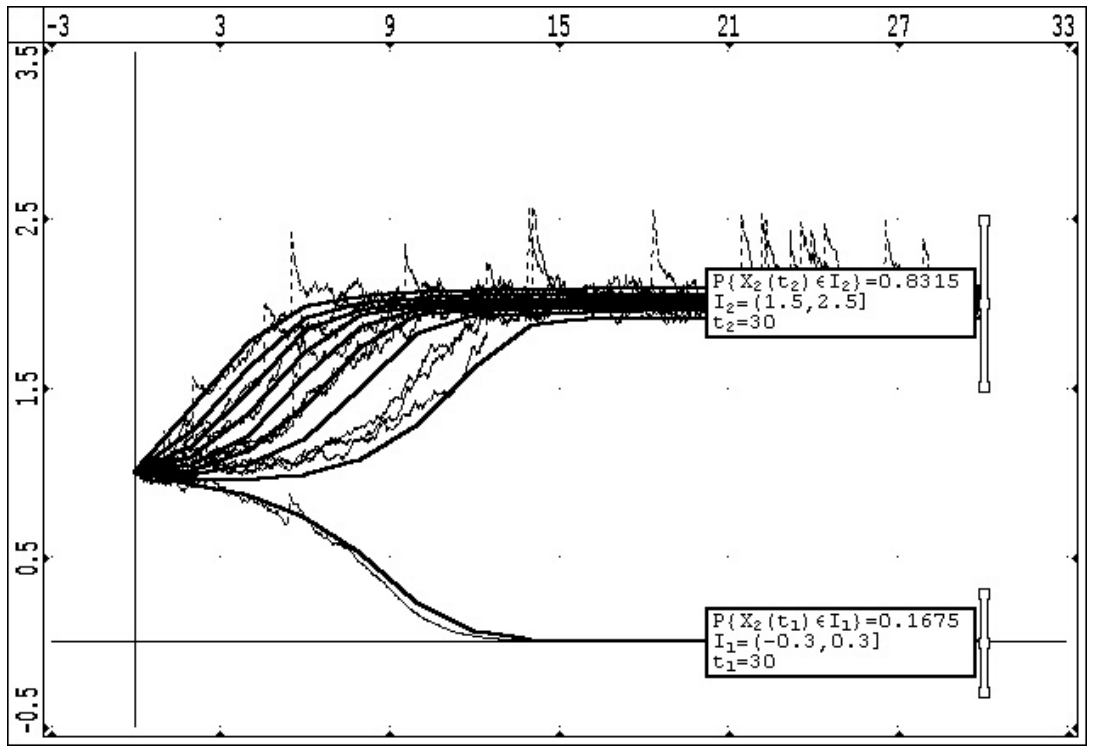

Fig. 2. Stochastic component $Y(t)$ of the solution to (21)

what means that in this model the probability of survival for the second species is about $83.2 \%$.

One can check that the situation of the first species is much better in Gaussian model, where $\sigma_{1}=\sigma_{2}=0, \nu_{1}=\nu_{2}=0$. In this case we obtain 


$$
\mathbb{P}\{X(t) \in[0.5,3.5]\}=\mathbb{P}\{Y(t) \in[-0.3,0.3]\} \approx 0.488, \quad \text { for } t \geq 30
$$

what means that in this model the probability of winning for the first species is about $51.2 \%$.

Figures 1 and 2 represent both components of the solution $(X(t), Y(t))$ to the system of SDEs (21), for $t \in[0,30]$.

They are visualized with the help of 20 exemplary trajectories, 9 quantile lines, and 2 cylindrical probability measures, which were computed after producing 2000 trajectories of the solution.

We can see the influence of the Poisson random measure on the solution $(X(t), Y(t))$.

The set of 9 quantile lines (deciles) helps to follow time dynamics of the modelled event.

\section{References}

1. Embrechts P., Klüppelberg C., Mikosch T.: Modelling Extremal Events. SpringerVerlag, Berlin (1997)

2. Janicki, A.: Computer Simulation of Diffusions Driven by $\alpha$-stable Lévy Processes. Mathematics Comp. Simul. 38 (1995) 97-101

3. Janicki, A., Weron, A.: Simulation and Chaotic Behavior of $\alpha$-stable Stochastic Processes. Marcel Dekker, New York $(1994,2000)$

4. Janicki, A., Izydorczyk, A.: Computer Methods in Stochastic Modeling (in Polish). Wydawnictwa Naukowo-Techniczne, Warszawa (2001)

5. Gradalski, P.: Computer Construction of Solutions to Stochastic Differential Equations Driven by Lévy Processes (M.S. Thesis in Polish). Wrocław University of Technology, Wrocław (2001)

6. Janicki, A., Krajna, L.: Malliavin Calculus in Construction of Hedging Portfolios for the Heston Model of a Financial Market. Demonstratio Mathematica XXXIV (2001) 483-495

7. Kloeden, P.E., Platen, E.: Numerical Solution of Stochastic Differential Equations. Springer-Verlag, New York $(1992,1996,1998)$

8. Protter, P.: Stochastic Integration and Differential Equations - A New Approach. Springer-Verlag, New York (1990)

9. Sahni, S.: Data Structures, Algorithms and Applications in C++. WCB McGrawHill, Boston (1998)

10. Swan, T.: Mastering Windows Programming with Borland C++. SAMS Publishing, Indianapolis (1994)

11. Thompson J.R.: Simulation, A Modeler's Approach. J. Wiley \& Sons, London (2000) 